\section{Colonic stricture following conservative treatment of a colonoscopic perforation}

Colonic perforation is a well-known, leading complication of colonoscopy. The incidence of colonic perforation ranges from $0.03 \%$ to $0.65 \%$ in diagnostic colonoscopies and from $0.07 \%$ to $2.14 \%$ in therapeutic colonoscopies $[1,2]$.

A 66-year-old woman was admitted for left colonic stricture. This patient had been treated for colonic perforation almost 1 year previously. At that time, she had been undergoing investigation by the gastroenterology department because of intermittent diarrhea. Colonoscopy was otherwise unremarkable with completely normal colorectal findings. The patient developed abdominal pain, nausea, and slight abdominal distension a few hours after colonoscopy. Plain abdominal radiography showed free air under the right diaphragm. Nasogastric decompression, intravenous fluids, and antibiotic treatment were immediately started. The physical examination findings improved gradually. The nasogastric tube was removed on day 5 . A computed tomography scan was performed, which showed a normal abdominal image, and the patient was discharged on day 12 .
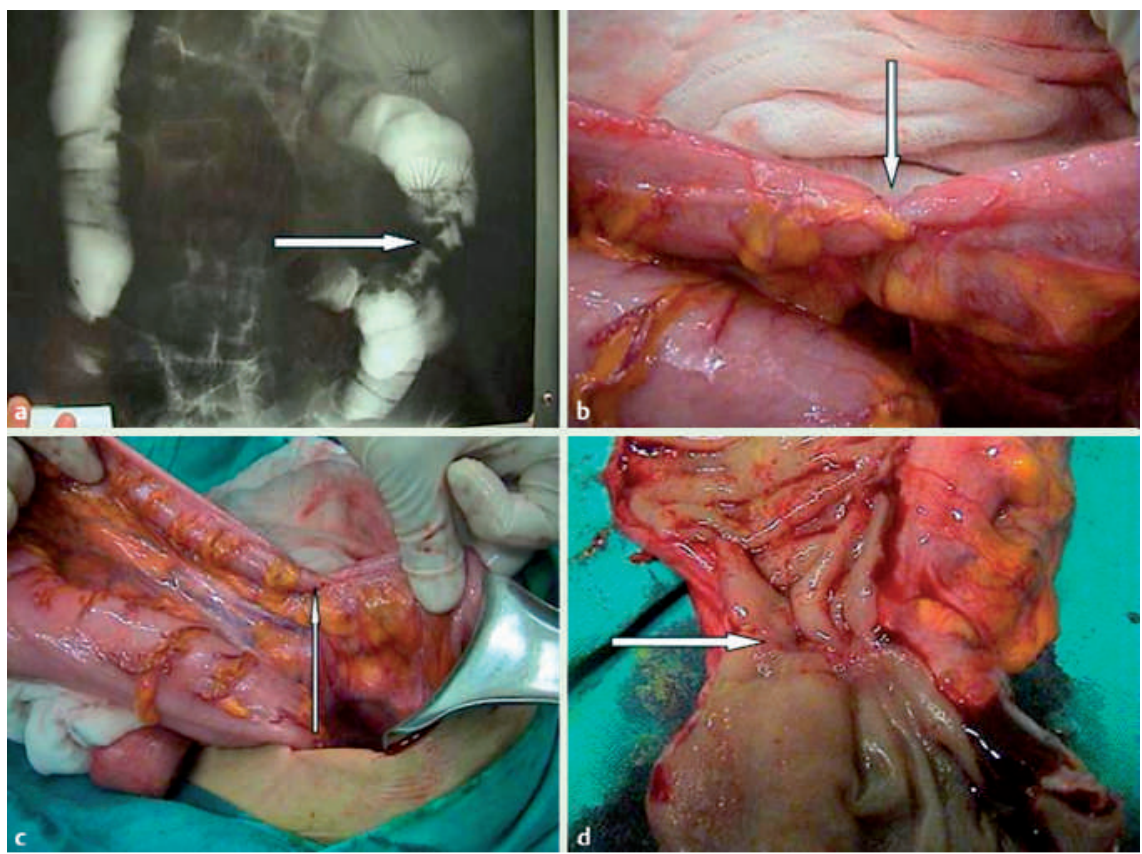

Fig. 1 a The stricture on the sigmoid junction with inadequate passage of the contrast to the proximal segments (arrow). b, c, $\mathbf{d}$ Intraoperative view: a thin benign-looking stricture that resembled an anastomotic stenosis was found on the very proximal sigmoid colon (arrow). complications of colonoscopy, such as splenic rupture [3] or transverse mesocolon laceration [4].

Endoscopy_UCTN_Code_CPL_1AJ_2AB

M.Akin ${ }^{1}$, B.Ege ${ }^{1}$, F.E.Akin ${ }^{2}$,

S.Leventoglu ${ }^{1}$, O.Kurukahvecioglu ${ }^{1}$,

K.Yusifzade ${ }^{1}$, B.B.Mentes ${ }^{1}$

1 Department of General Surgery, Gazi

University of Medical School, Ankara, Turkey

2 Department of Gastroenterology, Ankara Ataturk Research and Education Hospital, Ankara, Turkey

\section{References}

1 Damore LJ $2^{\text {nd }}$, Rantis PC, Vernava AM3 ${ }^{\text {rd }}$ et al. Colonoscopic perforation: etiology, diagnosis and management. Dis Colon Rectum 1996; 39: $1308-1314$

2 Kavic SM, Basson MD. Complications of endoscopy. Am J Surg 2001; 181: 319-332

3 Taylor FC, Frankl HD, Riemer KD. Late presentation of splenic trauma after routine colonoscopy. Am J Gastroenterol 1989; 84: $442-443$

4 Hernandez EJ, Ellington RT, Harford WV. Isolated transverse mesocolon laceration during routine colonoscopy. J Clin Gastroenterol 1999; 28: 46-48

Bibliography

DOI $10.1055 / \mathrm{s}-2007-995547$

Endoscopy 2008; 40: E89

(c) Georg Thieme Verlag KG Stuttgart · New York . ISSN 0013-726X

\section{Corresponding author}

M. Akin MD

Ayten sok.12/14 Mebusevler

Tandogan 06510

Ankara

Turkey

Fax: +90-312-2230528

makin@gazi.edu.tr 